\title{
Impact of the second wave of COVID-19 on cancer care at Birat Medical College and Teaching Hospital, Nepal
}

\author{
Sulav Sapkota ${ }^{1}$, Mona Priyadarshini ${ }^{2}$ \\ ${ }^{1}$ Assistant Professor, Department of Medical Oncology, ${ }^{2}$ Consultant, Department of Gynae-Oncology, Birat Medical \\ College Teaching Hospital, Biratnagar, Nepal
}

Background: The second wave of COVID-19 has plunged Nepal into a public health disaster and has also impacted cancer service to our department of Oncology. Aims and Objectives: To evaluate the impacts of COVID-19 second wave on outpatient department, inpatient department of oncology, Cancer screening, Cancer patients and Healthcare staffs of oncology department respectively. Materials and Methods: We did a prospective descriptive study from April 15, 2021 to June 142021 in our Oncology Department at Birat Medical College and Teaching Hospital. All the histopathologically proven cancer patients attending the oncology unit during the specific study periods were taken as study population. Proforma comprising patient details were maintained in excel sheet and the collected data were analyzed with the help of Statistical Package for Social Sciences. Results: We have 78\% decrement in outpatient visits and $30 \%$ decrement in admissions during the study period. We had $89 \%$ reduction in the newly diagnosed cases and total fourteen COVID positive cases. About $50 \%$ of the COVID-positive cases expired due to desaturation caused by COVID lung while $50 \%$ survived with the medical support. Majority of the infection was due to community spread. PCR test was advised for all the COVID suspects with features of fever and separate isolation ward was implemented for such patients. All of the healthcare staffs of oncology unit were immunized with vaccines as per the government protocol and none of them contracted COVID during the pandemic. Conclusion: Second wave of COVID-19 had a severe impact on cancer service at our Institution. With the decrement in COVID cases and ease of lockdown, slowly we are trying to gain our momentum with utmost COVID precautions.
Access this article online

\section{Website:}

http://nepjol.info/index.php/AJMS DOI: 10.3126/ajms.v13i2.42069

E-ISSN: 2091-0576

P-ISSN: $2467-9100$

Copyright (c) 2022 Asian Journal of Medical Sciences

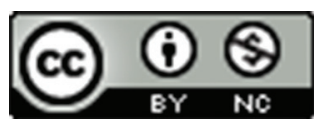

This work is licensed under a Creative Commons Attribution-NonCommercial 4.0 International License.

Key words: Cancer; Coronavirus disease; Nepal; Second wave

\section{INTRODUCTION}

The first wave of coronavirus disease 2019 (COVID-19) which is caused by severe acute respiratory syndrome coronavirus 2 (SARS-CoV-2), ${ }^{1}$ was declared pandemic ${ }^{2,3}$ on 11 March 2020. We had a negative impact due to the first wave of COVID-19 on cancer management at our institution. ${ }^{4}$ With the end of the first wave of COVID-19, lockdown was removed in Nepal and normal life was beginning by the end of 2020. However, government still implemented proper hand sanitization, use of mask as well as social distancing for COVID precaution. The second wave of COVID-19 cases in Nepal began to impact the country in April 2021 and has quickly become more widespread and severe than the first wave in 2020. According to data published by the Ministry of Health and Population (11 May), out of 18,400 swab tests, 9,271 cases were COVID positive with case positivity rate around $50 \%$ the highest rate across the globe at that moment. ${ }^{5}$ As we know Cancer patients are more prone to COVID-19 as they are more weak, immunosuppressed having multiple comorbidities. ${ }^{6}$ We have a well equipped inpatient oncology department (IPD) with trained sisters who deal with chemotherapy along with Medical Oncologist and Gyneoncologist. We have separate oncology outpatient department (OPD) for outpatient 
patients. We run a separate cancer screening OPD for breast and gynecological cancer screenings. Since there are less researches on the impact of second wave of COVID-19 on cancer patients in Nepal, hence in this study, we wanted to evaluate the impact of second wave of COVID-19 on the cancer service in our Oncology Department at our institution. This study will help us to implement plans and policies regarding managing cancer care in our institution in the future in case similar epidemic or pandemic is inevitable.

\section{Aims and objectives}

To evaluate the impacts of COVID-19 second wave on cancer care.

\section{MATERIALS AND METHODS}

This prospective descriptive study was conducted from April 15, 2021, to June 14, 2021, in the department of Oncology at Birat Medical College Teaching Hospital, Biratnagar, Nepal. The study was pre-approved by the departmental review committee for the permission. All the histopathologically proven cancer patients attending the oncology unit during the specific study periods were taken as study population. Those not giving consent for enrollment in the study were excluded. Polymerized chain reaction (PCR) positivity was taken as positivity of COVID-19 test. Impact of second wave of COVID-19 was studied on different grounds such as outpatient department service, Inpatient department service, Health of cancer patients as well as healthcare workers, respectively. Proforma comprising patient details including age, sex, associated comorbidities, types of cancer, treatment received, COVID test, result, etc were maintained in excel sheet and the collected data were analyzed with the help of Statistical Package for Social Sciences. The main objective of the study was to calculate the decrement in total visits of the patients, decrement in total number of patients and decrement in the diagnosis of new cases. COVID status was catagorizd as mild, moderate, and severe. Mild cases were who for home isolation without oxygen, moderate cases who required oxygen support during admission to hospital and severe cases were those who required mechanical ventilation support. COVID duration was considered from the day of PCR test positivity to the day patient got better and discharged or patient expired. The patients who were taking full treatment at our institution were considered as booked cases. Travel history especially for the treatment to India was taken from the patients as the second wave of COVID-19 in Nepal followed after the second wave in India. The study was supported by a dedicated team comprising of medical oncology team, enrolled patient along with family involvement. Data was collected by either real time meeting, virtual meeting or tele-communication. Real-time meeting was done as faceto-face interview of about 15-20 min at OPD or IPD and the data were collected into the excel sheet. Written consent was obtained from the patient for the treatment as well as research purpose.

\section{RESULTS}

\section{Impact on OPD service}

With the surge in second wave of COVID-19, initially, we had moderate decrement in the patient flow. There were total of 129 OPD visits with $78 \%$ decrement in OPD visit and $89 \%$ decrement in the diagnosis of new cases (Table 1). However during the mid May, COVID-19 was at the peak and OPD service was closed for a month in view of risk of COVID transmission. During the closure, only emergency patients were seen through emergency department and were admitted to the inpatient department for chemotherapy or supportive care. OPD service resumed after June 152021.

\section{Impact on IPD service}

Similar to OPD service, IPD service was hit hardly by COVID-19 second phase. Whole IPD department was converted into the COVID department and oncology was shifted back to Medicine ward semi deluxe cabin for about 2 months. Since the hospital was giving service to COVID patients too, there was curtailment of oncology sisters to help for COVID care. Almost $50 \%$ of sisters were transferred to COVID care and remaining were taking care of cancer patients as well as medical patients. All the admission for chemotherapy were done through the emergency department after screening properly. Incase of suspected COVID, patients were screened at the Isolation ward before the negativity of PCR test. If the PCR test was found to be positive, patients were transferred to COVID care department. The standard chemotherapy regimen protocol was followed with some modifications. Weekly regimen were converted to thrice weekly regimen in order to decrease the frequent hospital visits. Elderly patients were given relaxation in duration of chemotherapy and few were treated with metronomic oral therapy to overcome the

\begin{tabular}{lcc} 
Table 1: Outpatient department service & \\
\hline Characteristics & $\begin{array}{c}\text { Frequency } \\
\text { (N) }\end{array}$ & Percentage \\
\hline Total number of visits & 129 & - \\
Total number of patients & 48 & - \\
Male patients & 22 & 45 \\
Female patients & 26 & 55 \\
New patients & 11 & 23 \\
Old patients & 37 & 77 \\
Decrement in visits & - & 78 \\
Decrement in patients & - & 75 \\
Decrement in new diagnosis case & - & 89 \\
\hline
\end{tabular}


pandemic duration. However, hematological malignancies followed the treatment guidelines strictly than the solid malignancy patients. Majority of the patients receiving chemotherapy were discharged on the same day. There were total ninety-seven admissions during the study period (Table 2) with more than $90 \%$ of old patients admission. There was 30\% decrement in the total admission and 25\% decrement in the admission of new cases. The patients and visitors were advised to wear proper mask, gloves, faceshield, and avoid crowds. There was no shortage of chemotherapy drugs due to lockdown implemented by the government. We implemented The European Society of Medical Oncology approach for categorizing patients into different priorities for receiving cancer therapy during the second wave too. ${ }^{7}$

\section{Impact on cancer screening service}

During the $1^{\text {st }}$ month of the study, there was about $71 \%$ decrement in the cancer screening of breast cancer as well as gynecological cancers (Table 3). Mammogram was used to screen for breast cancer while pap smear, colposcopy, biopsy were used to screen for the gynecological cancers. Screening program was closed during the $2^{\text {nd }}$ month in view of risk of COVID transmission. There was decrement in the diagnosis of new cases by about $48 \%$ by screening during the study which included mainly gynecological cancers.

\section{Impact on cancer patients}

The second wave of COVID-19 impacted cancer patients hardly than the first wave. Total fourteen patients contracted COVID-19 during the study period among which seven succumbed to death and only seven could

\begin{tabular}{lcc} 
Table 2: Inpatient department service & \\
\hline Characteristics & Frequency (N) & Percentage \\
\hline Total number of admissions & 97 & - \\
Total number of patients & 64 & - \\
Male patients & 32 & 50 \\
Female patients & 32 & 50 \\
New patients & 6 & 10 \\
Old patients & 58 & 90 \\
Decrement in admissions & - & 30 \\
Decrement in patients & - & 26 \\
Decrement in new cases & - & 25 \\
\hline
\end{tabular}

\begin{tabular}{lcc} 
Table 3: Cancer screening service & \\
\hline Characteristics & $\begin{array}{c}\text { Frequency } \\
\text { (N) }\end{array}$ & Percentage \\
\hline Total number of screening & 25 & - \\
Breast cancer screening & 6 & 24 \\
Gynaecological cancer screening & 19 & 76 \\
New patients & 12 & 48 \\
Decrement in screening & - & 71 \\
Decrement in new diagnosis & - & 52 \\
case & & \\
\hline
\end{tabular}

manage to survive with the help of medical supports (Tables 4 and 5). The age range of patients who expired was from 20 to 92 -years-old. Majority of the positive cases were Lung cancer (29\%) and Hematological cancers $(29 \%)$. There were only $4(29 \%)$ booked cases from our institution and only $3(21 \%)$ cases had travel history to India for the treatment of cancer. All the positive cases were transferred to COVID centers and were admitted to the Intensive Care Unit due to oxygen desaturation. Mortality was due to pneumonia caused by corona virus known as COVID lung. Out of seven mortality, only four cases were in advanced stage undergoing treatment who had severe immunosuppression due to chemotherapy resulting in their death with mortality index of about $57 \%$.

\section{Impact on healthcare staffs}

Healthcare staffs including doctors, nursing staffs, helpers were all in the fear of contracting coronavirus while working in our department. Handwashing, face mask, hand sanitization, and proper gown were implemented strictly during the working hours. Fifty percent of nursing staffs were transferred to COVID care where they use to work for 14 days continuously and isolate for 14 days. Rest fifty percent nursing staffs were implemented to takecare of cancer patients as well as medical patients. All the staffs were regularly screened with PCR test on the basis of symptoms. However, none of the healthcare staffs contracted COVID during the study period. Our hospital has implemented government insurance program for all the healthcare staffs. Besides it, all the healthcare staffs were vaccinated twice for COVID-19 as per the government protocol. All the academics and research-oriented meetings were conducted on virtual platforms.

\section{DISCUSSION}

In our study, there was more decrement $(78 \%)$ in OPD visits than the IPD admission (30\%) which was primarily due to lockdown implemented by the government to prevent the transmission of COVID and IPD patients were mainly the old patients $(90 \%)$ who were continuing the chemotherapy with all the precautions. However, the worst scenario is the $89 \%$ decrement in the new cancer cases by OPD visit as it will delay the diagnosis of patients resulting in more advanced stages of cancer increasing the morbidity as well as mortality of the patients. ${ }^{8,9}$ This decrement in new diagnosis is probably caused by fear of the patients to visit hospitals where the virus is present. ${ }^{10}$

Ensuring safety of cancer patients along with the healthcare workers during this second wave of COVID was of prime importance. However, we could not have utmost control in our hands. Even with the COVID precautions and proper 
Table 4: Characteristics of cancer patients succumbed to COVID second wave

\begin{tabular}{|c|c|c|c|c|c|c|c|}
\hline Number of Cases & 1 & 2 & 3 & 4 & 5 & 6 & 7 \\
\hline Sex & Female & Male & Male & Male & Female & Female & Male \\
\hline Age & 29 & 20 & 55 & 92 & 67 & 65 & 64 \\
\hline Marital status & Yes & No & Yes & Yes & Yes & Yes & Yes \\
\hline \multirow[t]{2}{*}{ Diagnosis } & Ovary & Lymphoma & Lung & Prostate & Lung & CLL & Brain \\
\hline & Stage 2 & Stage 4 & Stage 4 & Stage 2 & Stage 4 & & Stage 4 \\
\hline Disease status & Remission & Advanced stage & Advanced & Remission & Advanced & Remission & Advanced \\
\hline COVID status & Severe & Moderate & Severe & Severe & Moderate & Severe & Moderate \\
\hline COVID Duration & 3 days & 30 days & 8 days & 6 days & 5 days & 12 days & 30 days \\
\hline Booked cases & Unbook & Unbook & Unbook & Booked & Unbooked & Booked & Unbooked \\
\hline Travel History & No & Yes & Yes & No & No & No & No \\
\hline
\end{tabular}

Table 5: Characteristics of cancer patients survived to COVID second wave

\begin{tabular}{|c|c|c|c|c|c|c|c|}
\hline Number of cases & 1 & 2 & 3 & 4 & 5 & 6 & 7 \\
\hline Sex & Female & Male & Female & Male & Male & Male & Male \\
\hline Age & 15 & 50 & 67 & 60 & 65 & 55 & 25 \\
\hline Marital status & No & Yes & Yes & Yes & Yes & Yes & No \\
\hline \multirow[t]{2}{*}{ Diagnosis } & MDS & Prostate & Breast & Lung & Lung & Colon & Lymphoma \\
\hline & & Stage 2 & Stage 3 & Stage 4 & Stage 4 & Stage 2 & Stage 3 \\
\hline Disease status & Remission & Remission & Advanced & Advanced & Advanced & Remission & Advanced \\
\hline COVID status & Moderate & Moderate & Moderate & Moderate & Moderate & Moderate & Moderate \\
\hline COVID Duration & 14 days & 16 days & 21 days & 12 days & 14 days & 16 days & 13 days \\
\hline Booked cases & Booked & Unbooked & Unbooked & Unbooked & Unbooked & Booked & Unbooked \\
\hline Travel History & No & No & Yes & No & No & No & No \\
\hline
\end{tabular}

PPE, fourteen cancer patients contracted COVID during the second wave and seven cases succumbed to death. Out of the fourteen positive cases, four cases were our booked cases while rest ten were the unbooked cases. Among the booked cases, two patients succumbed to death while two managed to survive the COVID. The worst part is that the three patients who died due to COVID were in clinical remission to cancer and only four patients were advanced cases undergoing treatment. This resulted in the effort to cure the cancer futile.

Only three positive cases had the travel history to India where they contracted the COVID and rest of the eleven cases did not have the travel history. This emphasizes that the majority of the patients $(79 \%)$ had community spread of COVID which reveals the severity of the pandemic in our eastern province of Nepal. Though the government had reinforced sealing of international borders to India as well as canceled the international flights, it could not confine second wave of COVID to have community spread on time. ${ }^{11}$ The cause could be due to mutation of the COVID-19 resulting in aggressive strain that resulted community spread in no time. ${ }^{12}$

Four $(29 \%)$ positive cases were lung cancer patients and four $(29 \%)$ were blood cancer patients. COVID was more common to them, cause could be lung cancer patients have physiological as well as pathological changes in their lungs resulting in severe infection. ${ }^{13}$ Blood cancer patients may be due to their decrease cellular as well as humoral immunity were more prone to get the severe infection. ${ }^{14}$ These patients needed more intensive care supports and high risk of mortality. ${ }^{15}$

Our institution had a great role play in preventing as well as treating COVID patients during the second wave. Awareness programs on COVID-19 symptoms, use of personal protective equipments by health care workers, screenings tests, and quarantine/isolation protocols was implemented by the hospital. Full fledge COVID Hospital was started to treat COVID-positive patients in our hospital. All the patients were screened for COVID before admission into the oncology department on regular basis. Even the medical staffs were screened on regular basis and quarantined as required. Handwashing, face mask, and sanitization were implemented strictly by the hospital management. Even the insurance program and vaccination was provided to all the Healthcare staffs by the hospital. None of the oncological health care staffs contracted COVID during the second wave which could be due to handwashing, hand sanitization and proper facemask implementation by the oncology department.

\section{Limitations of the study}

This is a single center study with smaller study population and comparison inbetween the impacts of the first wave and second wave of COVID 19 is not being studied. 


\section{CONCLUSION}

AltThough we have a severe impact due to second wave of COVID-19 on cancer care at our institution, with the support of all, we tried to provide the best possible care even in these hard times. Vaccination program is ongoing and we hope for the control of the COVID-19 pandemic to provide better cancer care in the future.

\section{ACKNOWLEDGMENT}

The author would like to acknowledge all the patients and healthcare staffs involved in the Medical Oncology department of Birat Medical College, Biratnagar during the study period.

\section{REFERENCES}

1. Available from: https://www.who.int/docs/default-source/ coronaviruse/situation-reports/20200221-sitrep-32-covid-19.pdf

2. Available from: https://www.pharmaceutical-technology.com/ news/who-declares-covid-19-pandemic

3. Chan JF, Yuan S, Kok KH, To KK, Chu H, Yang J, et al. A familial cluster of pneumonia associated with the 2019 novel coronavirus indicating person-to-person transmission: A study of a family cluster. Lancet. 2020;395(10223):514-523.

https://doi.org/10.1016/S0140-6736(20)30154-9

4. Sapkota $S$ and Priyadarshini M. Impact of the COVID-19 on cancer management at Birat Medical College and Teaching Hospital, Nepal. Asian J Med Sci. 2021;12(5):21-24.

https://doi.org/10.3126/ajms.v12i5.34024

5. Available from: https://www.inf.org.uk/coronavirus-a-secondwave-of-covid-19-in-nepal

6. Zhang L, Zhu F, Xie L, Wang C, Wang J, Chen R, et al.
Clinical characteristics of COVID-19-infected cancer patients: A retrospective case study in three hospitals within Wuhan, China. Ann Oncol. 2020;31(7):894-901.

https://doi.org/10.1016/j.annonc.2020.03.296

7. Available from: https://www.esmo.org/guidelines/cancerpatient-management-during-the-covid-19-pandemic

8. Huillard $O$ and Goldwasser F. Cancer treatment during the Coronavirus disease 2019 pandemic: Do not postpone but decide wisely. Eur J Cancer. 2020;135:51. https://doi.org/10.1016/j.ejca.2020.05.024

9. Manso L, de Velasco $G$ and Paz-Ares L. Impact of the COVID-19 outbreak on cancer patient flow and management: Experience from a large university hospital in Spain. ESMO Open. 2020;5 Suppl 2:e000828.

https://doi.org/10.1136/esmoopen-2020-000828

10. Rogado J, Obispo B, Gullón P and Lara MÁ. Impact of the COVID-19 pandemic in cancer diagnosis in the first and second waves in one of the most affected cancer areas in the city of Madrid (Spain). Int J Cancer. 2021;148(7):1794-1795. https://doi.org/10.1002/ijc.33462

11. Available from: https://www.reliefweb.int/report/nepal/nepalfacing-unprecedented-second-covid-wave-care-supportsvulnerable-communities

12. Available from: https://www.inf.org/news/tough-times-posed-bythe-second-wave-of-epidemic

13. Passaro A, Bestvina C, Velez Velez M, Garassino MC, Garon E and Peters S. Severity of COVID-19 in patients with lung cancer: Evidence and challenges. J Immunother Cancer. 2021;9(3):e002266.

https://doi.org/10.1136/jitc-2020-002266

14. Available from: https://www.healio.com/news/hematologyoncology/20210217/covid19-and-blood-cancer-evolvingscience-on-a-dangerous-comorbidity

15. Lee LYW, Cazier JB, Starkey T, Briggs SE, Arnold R, Bisht V, et al. COVID-19 prevalence and mortality in patients with cancer and the effect of primary tumour subtype and patient demographics: A prospective cohort study. Lancet Oncol. 2020;21(10):1309-1316. https://doi.org/10.1016/S1470-2045(20)30442-3

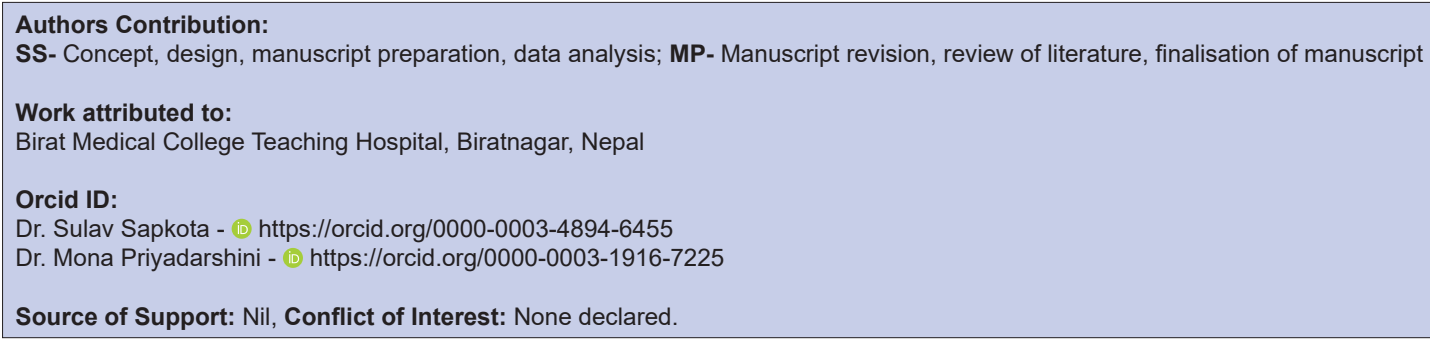

